\title{
Shape oscillations of a nonspherical water droplet
}

\author{
Mounika Balla, ${ }^{1}$ Manoj Kumar Tripathi, ${ }^{2}$ and Kirti Chandra Sahu ${ }^{1, *}$ \\ ${ }^{1}$ Department of Chemical Engineering, Indian Institute of Technology Hyderabad, Sangareddy 502 285, Telangana, India \\ ${ }^{2}$ Indian Institute of Science Education and Research, Bhopal 462 066, Madhya Pradesh, India
}

(Received 21 August 2018; revised manuscript received 11 January 2019; published 11 February 2019)

\begin{abstract}
The dynamics of a nonspherical droplet falling in air is investigated via three-dimensional numerical simulations of the Navier-Stokes and continuity equations. The main focus of this work is to study the effects of the relative influence of surface tension force over the inertial force and the orientation of the droplet. The values of the dimensionless parameters considered in this study are similar to those of a typical falling raindrop. Our results obtained from the numerical simulations for low values of Galilei number agree well with the theoretical predictions in the microgravity condition and in the limit of creeping flow. However, in the inertia-dominated region and for high initial aspect ratio, the droplet undergoes predominant nonlinear oscillations and subsequently breaks up. To the best of our knowledge, none of the previous studies have investigated dynamics of a tilted drop and observed the behavior reported in the present study.
\end{abstract}

DOI: 10.1103/PhysRevE.99.023107

\section{INTRODUCTION}

A variety of shapes are observed in falling raindrops, as reported by several authors previously [1-7]. This is mainly due to the interplay between the gravitational and the surface tension forces. As a matter of course, there are other effects, such as wind, temperature and humidity gradients, presence of aerosols, ground topology, etc., which influence the dynamics of raindrops; the effects of these parameters are neglected in the present study. The main focus of the present study is only to investigate the shape oscillations of a falling raindrop under the influence of surface tension and gravity.

During precipitation, the size of a raindrop increases as it coalesces with other raindrops, and gets fragmented into smaller droplets when it becomes big (diameter, $d \approx 6-8 \mathrm{~mm}$ ) [5]. The studies of Pruppacher and coworkers $[1,8]$ reveal that small droplets $(d<1 \mathrm{~mm})$ are spherical, but bigger raindrops $(d>1 \mathrm{~mm})$ are nonspherical and become oblate or prolate or form more complex shapes [3]. A small spherical raindrop (when the surface tension force is high) attains a terminal velocity and its equilibrium shape when the drag force acting in the upward direction balances its weight. However, for large raindrops, the surface-tension force is low, and they tend to deform and undergo shape oscillations. Several models were developed, see, for instance, Refs. [1,9-11], to predict the shape oscillations of a raindrop in different conditions.

Rayleigh [12] was the first to investigate shape oscillations of a droplet in viscous dominated and inviscid regimes separately. Feng and Beard [13] extended this theoretical analysis to study nonspherical droplets observed in atmospheric conditions and derived the expressions of frequencies for general oscillation modes. Szakall et al. [3] studied the shape oscillations of raindrops of different sizes experimentally and compared them with the theoretical predictions of Feng and

*Corresponding author: ksahu@iith.ac.in
Beard [13]. Recently, Agarwal et al. [14] investigated the dynamics of a nonspherical liquid droplet falling in air for high inertia by conducting direct numerical simulations of the Navier-Stokes equations. They found that when a droplet starts to fall from an oblate or prolate shape, it undergoes oblate-prolate oscillations, whose amplitude decreases slowly. However, in the study of Agarwal et al. [14], the shape oscillations were restricted only in the flow and orthogonal to the flow directions. As they attempted to find the path instability of a falling droplet (wobbling motion), the inertia was varied for a fixed value of the surface tension. The parameter values considered in their study are in the range for which a rising air bubble undergoes wobbling motion.

Shape oscillations of a droplet in liquid-liquid systems were also studied by several researchers [15-17] due to its relevance in many practical applications. Koh and Leal $[15,16]$ observed that a liquid drop with a small degree of nonsphericity comes back to a spherical shape as it falls, but a drop with a large degree of nonsphericity, continues to elongate with time and breaks up from its tail. Edge and Grant [18] conducted experiments on droplet of an organic liquid moving in water due to gravity and found that small droplets move in a straight trajectory, whereas big ones undergo wobbling motion. Miller et al. [19] derived an analytical expression to obtain frequency of shape oscillations of a droplet in a liquid-liquid system and found that viscous dissipation and velocity distribution are responsible for the damping oscillations in case of low- and high-viscosity ratios between the continuous and dispersed phases, respectively.

In the present study, we investigate dynamics and shape oscillations of a nonspherical raindrop (of equivalent spherical radius, $R_{\text {eq }}$ ) by conducting three-dimensional (3D) numerical simulations. The emphasis has been given to the effect of the relative influence of the surface tension force over the inertial force and the orientation of the droplet. The numerically obtained frequency and decay rate of an oscillating droplet are compared against the theoretical predictions of Lamb [9] 
and Tsamopoulos and Brown [10] performed in the limit of creeping flow and microgravity conditions. The dimensionless numbers are calculated using the properties of air and water, such that the density ratio is $\left.\rho_{r}\left[\equiv \rho_{i} / \rho_{o}\right)\right]=998$ and the viscosity ratio is $\left.\mu_{r}\left[\equiv \mu_{i} / \mu_{o}\right)\right]=55$. Here $\rho_{i}, \mu_{i}$ and $\rho_{o}, \mu_{o}$ are the density and dynamic viscosity of the dispersed (water) and continuous (air) phases, respectively. The value of the interfacial tension $(\sigma)$ of the air-water interface is taken to be $72 \mathrm{mN} / \mathrm{m}$. For typical raindrops of radii 1 to $6 \mathrm{~mm}$, the Galilei number $\left[\mathrm{Ga}\left(\equiv \rho_{o} g^{1 / 2} R_{\mathrm{eq}}{ }^{3 / 2} / \mu_{o}\right)\right]$ varies from 10 to 100 , and the Eötvös number $\left[\mathrm{Eo}\left(\equiv \rho_{o} g R_{\mathrm{eq}}{ }^{2} / \sigma\right)\right]$ varies from 0.001 to 0.005 . Increasing $\mathrm{Ga}$ decreases the influence of the viscous force over the gravitational force, whereas increasing Eo signifies the dominance of the gravitational force over the surface tension force. Therefore, the same range of the parameter values are considered in the present study. We ask and attempt to answer the following questions, which have motivated the present study: (i) How is the dynamics of a tilted drop different from a normal prolate drop, whose major axis lies in the same direction as that of gravity, i.e., from our previous study [14]? (ii) How do the dynamics change with increasing aspect ratio of the droplet? (iii) For moderately high inertia, Agarwal et al. [14] observed some nonlinearity in shape oscillations. Does this lead to fragmentation if we increase the inertia further? (iv) What is the role of surface tension on the time period of oscillations of a tilted droplet.

Three-dimensional simulations are performed to answer the above-mentioned questions. The frequencies of shape oscillations obtained from our numerical simulations for different dimensionless parameters agree well with the theoretical prediction and also with the experimental result of Szakall et al. [3]. The amplitude of oscillations is found to be either constant or slightly increase with time for low and moderate inertia. However, as the inertia is increased further the droplet undergoes predominant nonlinear oscillations leading to breakup of the droplet. The droplet with high initial aspect ratio undergoes breakup even for low inertia. For a tilted droplet, it is observed that the vortex shedding patterns become asymmetrical, which is expected to influence the dynamics at a later stage. The shape oscillations and the associated vortex shedding may influence coalescence and breakup of raindrops. However, the interactions of multiple nonspherical droplets will be studied elsewhere.

The rest of this paper is organized as follows. The theoretical expressions for the frequency of oscillations and the decay rate are derived in Appendix. The problem formulation and the numerical method used are discussed in Sec. II. The results obtained from the study are presented in Sec. III. Finally, in Sec. IV, the concluding remarks are provided.

\section{FORMULATION}

The dynamics of an initially nonspherical liquid drop (as shown in Fig. 1) falling in air under the action of gravity $(g)$ is investigated via direct numerical simulations of three-dimensional incompressible Navier-Stokes and continuity equations. A Cartesian coordinate system $(x, y, z)$ is used with gravity $(g)$ acting in the negative $z$ direction. The aspect ratio, $A_{r}$, of the droplet is defined as $a / b$, where $a$ and $b$ are the diameters of the droplet at each time instance along

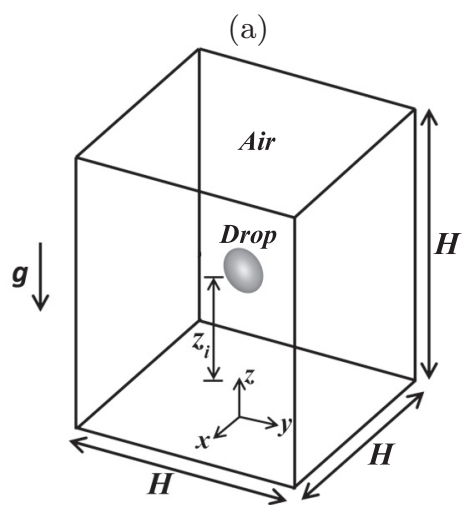

(b)

FIG. 1. (a) Schematic diagram of a nonspherical water droplet falling in air. A cubic computational domain of size $H \times H \times H$ is used and the bubble is placed at $\left(0,0, z_{i}\right)$. The values of $H=$ $75 R_{\mathrm{eq}}$ and $z_{i}=70 R_{\mathrm{eq}}$. The acceleration due to gravity $(g)$ acts in the negative $z$ direction. (b) The initial shape of a typical tilted droplet. Here $a$ and $b$ represent the diameters of the droplet along its initial major and minor axes, respectively. $\theta$ is the angle of the initial major axis of the droplet to the negative $y$ axis.

its initial major and minor axes, respectively. Thus, $A_{r}=1$ represents a spherical droplet. In Fig. 1, $\theta$ is the angle of the initial major axis of the droplet to the negative $y$ axis. The initial aspect ratio $\left(A_{r 0}\right)$ of the droplet is varied by keeping the volume constant, i.e., $4 \pi R_{\mathrm{eq}}^{3} / 3=\pi a^{2} b / 6$, wherein $R_{\mathrm{eq}}$ is the equivalent spherical radius of the droplet. Initially, both the droplet and surrounding air are stationary, and the droplet is placed at $z_{i}=70 R_{\text {eq }}$. In order to minimize the boundary effect, a sufficiently big computational domain of size $75 R_{\text {eq }} \times 75 R_{\text {eq }} \times 75 R_{\text {eq }}$ is considered in the present study. Free-slip and no-penetration conditions are imposed on all the boundaries of the computational domain. In order to refine the grid at the interface and in the regions of the domain where the gradients in velocity are large, we have used a wavelet error-based dynamic adaptive grid refinement feature incorporated in the Navier-Stokes solver Basilisk [20,21]. The smallest grid size, $\Delta\left(=H / 2^{\mathcal{L}}\right)$, can be obtained by specifying the refinement level, $\mathcal{L}$, in our computations.

\section{A. Governing equations}

The governing equations, which describe the dynamics of a falling liquid droplet in air, are the equations of mass and momentum conservation:

$$
\nabla \cdot \mathbf{u}=0
$$

$$
\begin{aligned}
\rho\left[\frac{\partial \mathbf{u}}{\partial \tau}+\mathbf{u} \cdot \nabla \mathbf{u}\right]= & -\nabla p+\nabla \cdot\left[\mu\left(\nabla \mathbf{u}+\nabla \mathbf{u}^{T}\right)\right] \\
& +\delta\left(\mathbf{x}-\mathbf{x}_{f}\right) \sigma \kappa \mathbf{n}-\rho g \mathbf{j} .
\end{aligned}
$$

Here $\mathbf{u}=(u, v, w)$ denotes the velocity field, where $u, v$, and $w$ represent the velocity components in the $x, y$, and $z$ directions, respectively; $p$ is the pressure field; $t$ denotes time; $\mathbf{j}$ denotes the unit vector along the vertical direction; $\delta\left(\mathbf{x}-\mathbf{x}_{f}\right)$ is the delta distribution function (denoted by $\delta$ hereafter) whose value is zero everywhere except at the interface, where 
$\mathbf{x}=\mathbf{x}_{f} ;$ and $\kappa(\equiv \nabla \cdot \mathbf{n})$ is the interfacial curvature, wherein $\mathbf{n}$ is the outward-pointing unit normal to the interface.

The interface separating the air and liquid phases is tracked by solving an advection equation for the volume fraction of the liquid phase, $c$ (such that, $c=0$ and 1 for the air and liquid phases, respectively):

$$
\frac{\partial c}{\partial t}+\mathbf{u} \cdot \nabla c=0
$$

The density, $\rho$, and the viscosity, $\mu$, are assumed to depend on $c$ as

$$
\begin{aligned}
& \rho=(1-c) \rho_{o}+c \rho_{i}, \\
& \mu=(1-c) \mu_{o}+c \mu_{i} .
\end{aligned}
$$

The following scaling is used to nondimensionalize the above governing equations:

$$
\begin{aligned}
(x, y, z) & =R_{\mathrm{eq}}(\tilde{x}, \tilde{y}, \widetilde{z}), \quad t=\widetilde{t} R_{\mathrm{eq}} / V, \mathbf{u}=V \tilde{\mathbf{u}}, \\
p & =\rho_{o} V^{2} \widetilde{p}, \quad \mu=\mu_{o} \tilde{\mu}, \quad \rho=\rho_{o} \tilde{\rho}, \delta=\tilde{\delta} / R_{\mathrm{eq}},
\end{aligned}
$$

where the velocity scale is $V=\sqrt{g R_{\mathrm{eq}}}$ and the tildes designate dimensionless quantities. After dropping tildes from all nondimensional variables, the governing dimensionless equations are given by

$$
\begin{gathered}
\nabla \cdot \mathbf{u}=0 \\
\frac{\partial \mathbf{u}}{\partial t}+\mathbf{u} \cdot \nabla \mathbf{u}=-\nabla p+\frac{1}{\mathrm{Ga}} \nabla \cdot\left[\mu\left(\nabla \mathbf{u}+\nabla \mathbf{u}^{T}\right)\right] \\
+\delta \frac{\nabla \cdot \mathbf{n}}{\mathrm{Eo}} \mathbf{n}-\rho \mathbf{j} .
\end{gathered}
$$

The dimensionless density and dynamic viscosity are given by

$$
\begin{aligned}
& \rho=(1-c)+c \rho_{r}, \\
& \mu=(1-c)+c \mu_{r} .
\end{aligned}
$$

The dimensionless frequency of oscillations and the decay rate are given by

$$
\begin{gathered}
\omega_{n}=\sqrt{n(n-1)(n+2) \frac{1}{\rho_{r} \mathrm{Eo}}}, \quad \text { and } \\
\alpha_{n}=(n-1)(2 n+1) \frac{\mu_{r}}{\rho_{r} \mathrm{Ga}}, \quad \text { respectively. }
\end{gathered}
$$

The reader has been referred to Appendix for the derivation of Eqs. (11) and (12). Note that for the inviscid case, $\alpha_{n}=0$ [12]. Like in the study of Szakall et al. [3], the fundamental $(n=2)$ mode is considered in the present study.

\section{B. Numerical method and grid convergence test}

A Navier-Stokes solver, Basilisk [20,21], has been used in the present study. A volume-of-fluid ( $\mathrm{VoF}$ ) method that incorporates a height-function-based balanced-force continuumsurface-force formulation for the inclusion of the surface force term in the Navier-Stokes equations is used. A dynamic adaptive grid refinement is incorporated, which provides large number of grid points or cells on the vortical and interfacial (a)

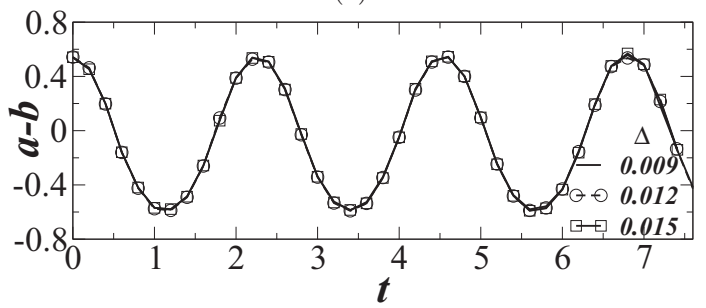

(b)

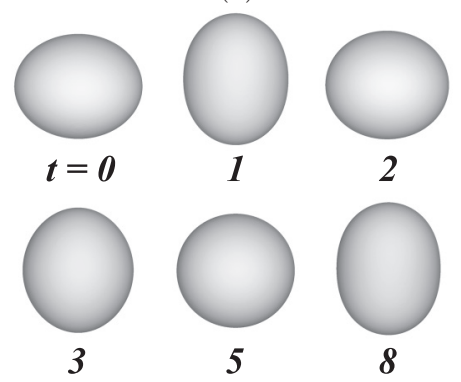

FIG. 2. (a) The temporal variations of $(a-b)$ obtained using $\Delta=0.009$ (solid line), $\Delta=0.012$ (circles), and $\Delta=0.015$ (squares). (b) Shapes of the droplet $(y-z$ view) at different time instants (which are indistinguishable for different grids considered). The remaining parameter values are $\mathrm{Ga}=100$, Eo $=10^{-3}, \theta=0$, and $A_{r 0}=1.331$. The red dotted line shows the aspect ratio of a perfect sphere.

regions. The solver has been validated extensively by comparing with previous experimental and computational studies (see, for instance, Refs. [22-24]). Here we have conducted a grid convergence test in Figs. 2(a) and 2(b), where the temporal variations of $(a-b)$ and shape evolutions of a droplet obtained using three different grids $(\Delta=0.009,0.012$, and 0.015 ) are plotted. It can be seen that the results obtained using different grids are indistinguishable, which confirms that the convergence with the grid is achieved. In view of this test, we use $\Delta=0.009$ to generate the rest of the results presented in this study.

\section{RESULTS AND DISCUSSION}

We begin the presentation of our results by comparing with the experiments of Szakall et al. [3] in Sec. III A.

\section{A. Comparison with Szakall et al. [3]}

As discussed in Sec. I, Szakall et al. [3] experimentally investigated the oscillations of a freely suspended water droplet $\left(R_{\text {eq }}=2.88 \mathrm{~mm}\right)$ inside a vertical wind tunnel. Air was injected in the upward direction with a velocity equal to the terminal velocity achieved by a droplet falling in atmospheric air under the action of gravity. Typical terminal velocities of raindrops of different sizes are given in Yau and Rogers [25] and Feng and Beard [13]. In our dimensionless formulation, the parameters considered by Szakall et al. [3] correspond to $\mathrm{Ga}=48.4$ and $\mathrm{Eo}=1.13 \times 10^{-3}$. Thus, in order to compare with the experimental results of Szakall et al. [3], we perform numerical simulations for $\mathrm{Ga}=48.4$ and $\mathrm{Eo}=1.13 \times 10^{-3}$ of initially oblate droplets $(\theta=0)$ with $A_{r 0}=1.331$ and 1.953 
(a)

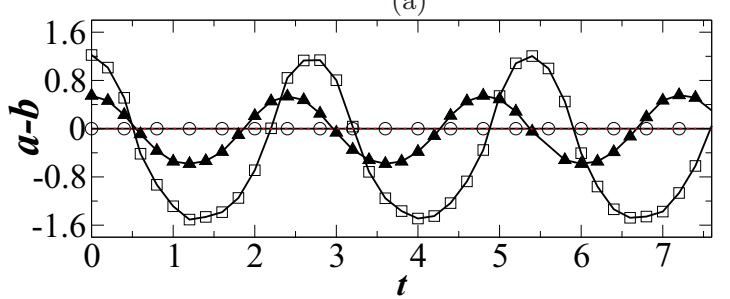

(b)

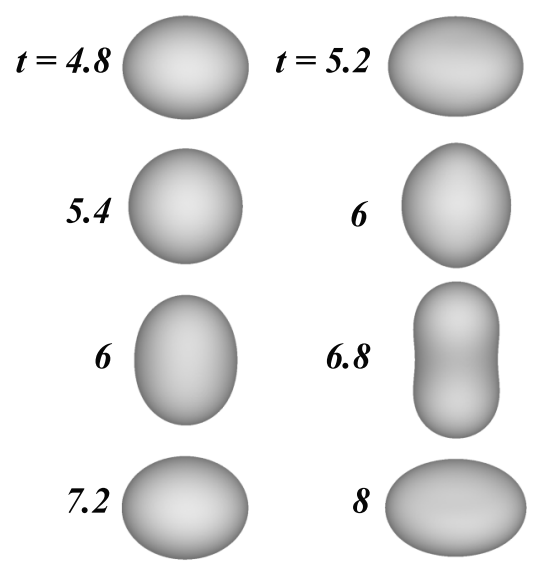

FIG. 3. (a) The temporal variations of $(a-b)$ for $A_{r 0}=1$ (circles), $A_{r 0}=1.331$ (filled triangles), and $A_{r 0}=1.953$ (squares). The red dotted line, which coincides with the result for $A_{r 0}=1$, shows the aspect ratio of a perfect sphere. (b) The evolutions of the shape of the droplet ( $y-z$ view) obtained for $A_{r 0}=1.331$ (left column) and 1.953 (right column). The remaining parameter values are $\mathrm{Ga}=$ 48.4, Eo $=1.13 \times 10^{-3}$, and $\theta=0$.

along with $A_{r 0}=1$ (initially spherical droplet). The variations of $(a-b)$ with time for different values of $A_{r 0}$ are plotted in Fig. 3(a). When the droplet starts to fall from a spherical shape $\left(A_{r 0}=1\right)$, it remains spherical, which is evident in Fig. 3(a) as $a-b \approx 0$ for the entire duration considered in the simulation [see line with circle symbols in Fig. 3(a)]. However, when the droplet starts from a nonspherical shape, it exhibits oblate-prolate-oblate shape oscillations via spherical shape [see the results for $A_{r 0}=1.331$ (filled triangles) and $A_{r 0}=1.953$ (squares) in Fig. 3(a)] due to inertia. In the inertia dominated region, this phenomenon was also found by Agarwal et al. [14], which was not observed in the creeping flow regime as considered by Koh and Leal $[15,16]$.

The dimensionless time period of the oscillations, $T_{p}$, for $A_{r 0}=1.331$ and 1.953 are found to be 2.42 and 2.72, respectively. The corresponding dimensionless time period of oscillations observed in the study of Szakall et al. [3] is $\approx 2.22$. Note that in Szakall et al. [3] the value of $A_{r 0}$ was not specified explicitly, and we have extracted this from their figure to be $\approx 1.4$. From Eqs. (11) and (12), the theoretically obtained dimensionless time period of oscillations $\left(2 \pi / \omega_{n}\right)$ and the decay rate $\left(\alpha_{n}\right)$ for this set of parameter values are 2.36 and 0.006 , respectively, which agree reasonably well with those obtained from our numerical simulations. It is also to be noted that as the value of $\alpha_{n}$ is small, the observed shape oscillations will take a very long time to decay, which is practically impossible to observe in case of a falling raindrop (a)

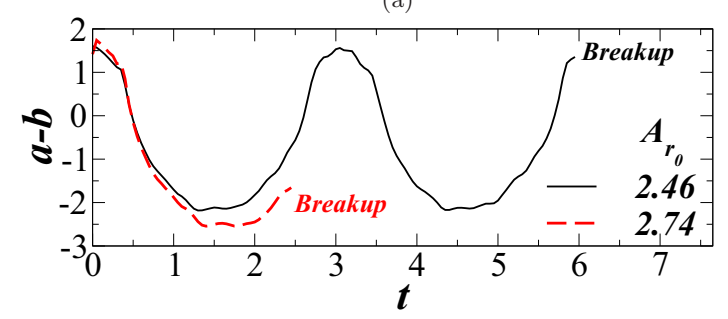

(b)

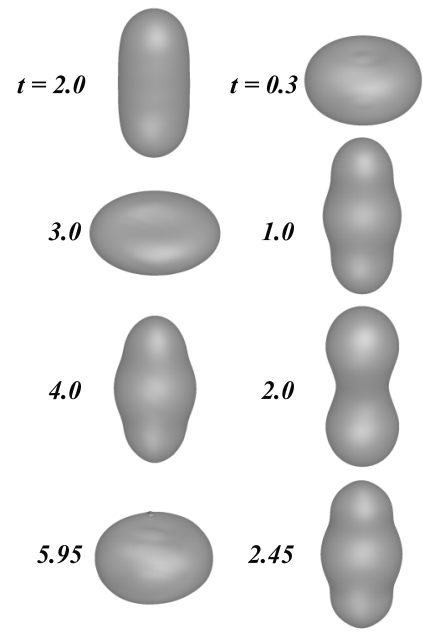

FIG. 4. (a) The temporal variations of $(a-b)$ for $A_{r 0}=2.46$ and 2.74. (b) The evolutions of the shape of the droplet obtained for $A_{r 0}=$ 2.46 (left column) and 2.74 (right column). The remaining parameter values are $\mathrm{Ga}=48.4$, $\mathrm{Eo}=1.13 \times 10^{-3}$, and $\theta=0$.

from clouds (altitudes 1 to $2 \mathrm{~km}$ ). Also during rain, these shape oscillations may influence coalescence and breakup phenomena of raindrops. However, the study of coalescence and breakup of raindrops is beyond the scope of the present study.

In Fig. 3(b), the evolutions of the droplet shape are presented for $A_{r 0}=1.331(4.8 \leqslant t \leqslant 7.2)$ and $1.953(5.2 \leqslant t \leqslant$ $8)$. As discussed above, the droplet remains spherical for $A_{r 0}=1$ (not shown). It can be seen that for $A_{r 0}=1.331$ (small degree of nonsphericity), the droplet undergoes oblateprolate shape deformation (via spherical shape) smoothly as seen in the left panel of Fig. 3(b), but we see some nonlinearlity for $A_{r 0}=1.953$ (high degree of nonsphericity) during the shape transition. This effect can be clearly seen in Fig. 3(b) at $t=6$ (nose shapes at top and bottom of the drop) and $t=6.8$ (capsule shape with curved sides). Agarwal et al. [14] also observed some nonlinearity for large initial deformation of the droplet. We observe that for $A_{r 0}>2$, the droplet undergoes violent shape oscillations and subsequent breakup. The shape oscillations prior to the breakup and the breakup points for $A_{r 0}=2.46$ and 2.74 are presented in Fig. 4(a). The corresponding shapes of the droplet for $A_{r 0}=2.46$ (left column) and 2.74 (right column) at different time before breakup are shown in Fig. 4(b). It can be seen the droplet with $A_{r 0}=2.74$ breaks earlier than the droplet with $A_{r 0}=2.46$.

In the rest of this study, we focus on the dynamics of a tilted water droplet falling in air. 


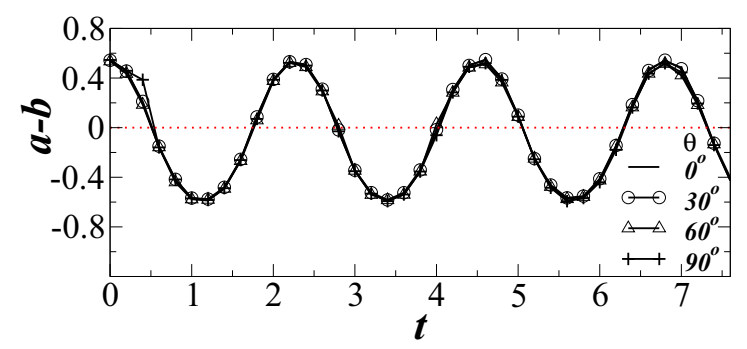

FIG. 5. Temporal variations of $(a-b)$ of the droplet for different values of initial tilt angle, $\theta$. The remaining parameter values are $\mathrm{Ga}=100$ and $\mathrm{Eo}=10^{-3}$. The red dotted line shows the aspect ratio of a perfect sphere.

\section{B. Tilted droplets}

In Fig. 5, the temporal variations of $(a-b)$ of a droplet starting from different orientations (i.e., different values of initial tilt angle, $\theta$ ) falling in air are shown. The values of the dimensionless parameters considered are $A_{r 0}=1.331$, $\mathrm{Ga}=100$, and Eo $=10^{-3}$. Although one would expect the dynamics to be different as we change the orientation of the droplet, the effect is not seen in the time period of the shape oscillations. The evolutions of droplet shape in the $y-z$ plane for $\theta=0^{\circ}, 30^{\circ}, 60^{\circ}$, and $90^{\circ}$ are shown in Fig. 6 . It can be seen that when a droplet starts from an initially deformed shape with $\theta=0$ (when the longer chord is in the horizontal direction) and $\theta=90^{\circ}$ (when the longer chord is in the vertical direction), the droplet undergoes oblate-sphericalprolate shape oscillations in the directions coinciding with the horizontal and the vertical directions. However, when the droplet starts from an orientation inclined to the horizontal (negative $y$ axis), the droplet undergoes shape oscillations in the direction of the initial inclination of the droplet and

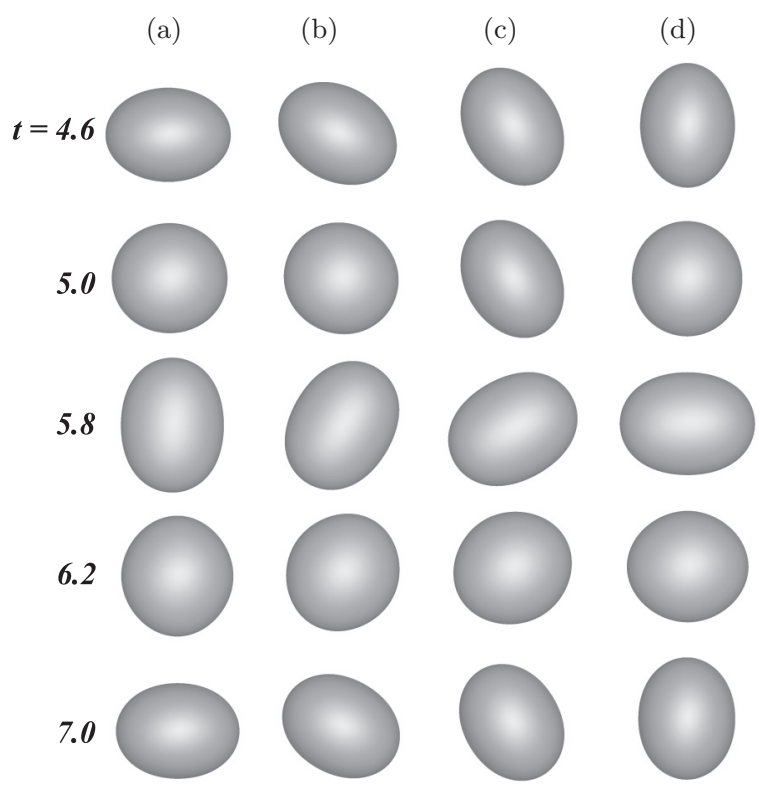

FIG. 6. Evolutions of the shape of the droplet ( $y-z$ view) for different tilt angles: (a) $\theta=0^{\circ}$, (b) $\theta=30^{\circ}$, (c) $\theta=60^{\circ}$, and (d) $\theta=90^{\circ}$. The remaining parameter values are $\mathrm{Ga}=100, \mathrm{Eo}=10^{-3}$, and $A_{r 0}=1.331$.

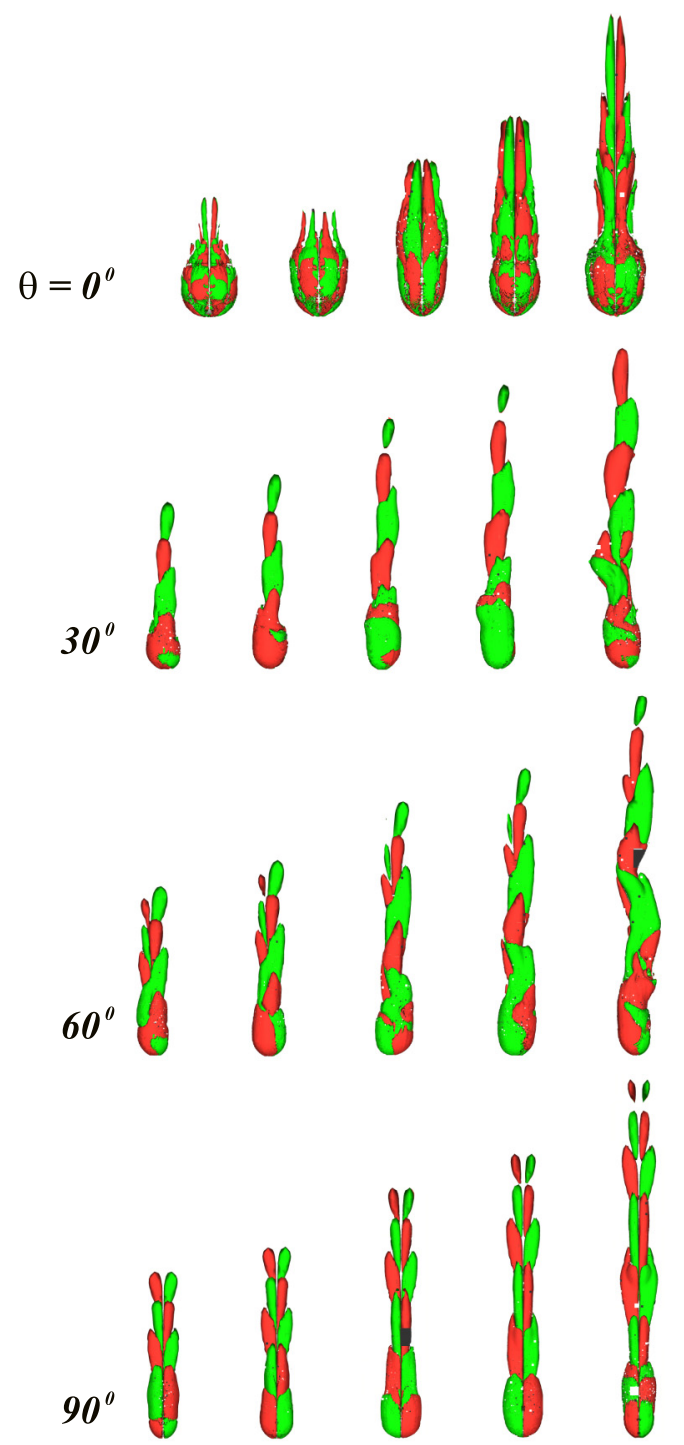

FIG. 7. Evolutions of the $y$ vorticity contours $\left(\omega_{y}= \pm 0.1\right)$ for different values of the tilt angle, $\theta$ (left to right for each value of $\theta: t=4.6,5,5.8,6.2$, and 7). The remaining parameter values are $\mathrm{Ga}=100$, Eo $=10^{-3}$, and $A_{r 0}=1.331$.

orthogonal to it [see Figs. 6(b) and 6(c)]. For low Ga value, at a later time, one would either expect the droplet to become spherical, as observed by Koh and Leal $[15,16]$, or to oscillate along the $z$ axis due to the gravitational effect and the hydrostatic pressure difference between the top and bottom of the droplet. However, this does not happen in the present case, as the inertia force dominates the dynamics.

Another interesting observation is presented in Fig. 7, where the evolutions of $z$ component of vorticity contours for different values of $\theta$ are shown. Symmetric vortex shedding (both clockwise and counterclockwise vortices shed at the same instant) is observed for $\theta=0^{\circ}$ and $90^{\circ}$, whereas the vortex shedding becomes countersymmetric (clockwise and counterclockwise vortices shed alternatively) for $\theta=30^{\circ}$ and $60^{\circ}$. The theoretical predictions of the dimensionless time period of oscillations $\left(2 \pi / \omega_{n}\right)$ and the decay rate $\left(\alpha_{n}\right)$ obtained from Eqs. (11) and (12) for this set of parameter values are 


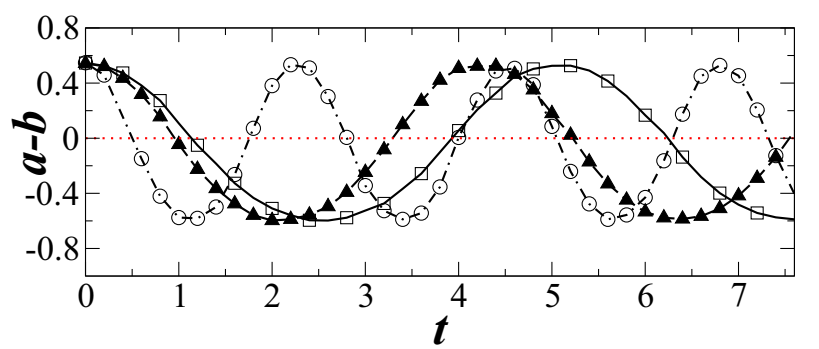

FIG. 8. The temporal variations of $(a-b)$ for $A_{r 0}=1.331$ $\left(\mathrm{Ga}=100\right.$ and $\left.\theta=45^{\circ}\right)$ for $\mathrm{Eo}=0.001$ (circles), 0.004 (filled triangles), and 0.005 (squares). The red dotted line shows the aspect ratio of a perfect sphere.

2.2 and 0.003 . The time period of oscillations obtained from our numerical computations is also $\approx 2.2$. A close inspection of Figs. 5 and 7 also reveals that the frequencies, $f$ associated with the shape oscillations and vortex shedding are about the same (i.e., $f=0.45$ ). Therefore, due to the synchronization of these frequencies, one would expect a significant effect on the droplet dynamics at later times. In spite of the vortex shedding in the wake region, we observe that a droplet migrates only in straight path. On the other hand, vortex shedding promotes wobbling motion in case of rising bubbles [22]. A similar observation on falling droplets was also reported by Saylor and Jones [7].

\section{Effects Eo and Ga}

Next we investigate the effects of Eo and Ga on the dynamics of a droplet with $\theta=45^{\circ}$. In Fig. 8, the variations of $(a-b)$ with time are shown for different values of Eo for $A_{r 0}=1.331$ and $\mathrm{Ga}=100$. It can be seen that increasing the value of Eo (decreasing the influence of surface tension over gravity) increases the time period of oscillations. This can be explained as follows. The dynamics is due to the interplay between inertia and surface tension forces. Inertia is responsible for the oblate-prolate shape oscillations. The surface tension tries to bring the droplet to a spherical shape.

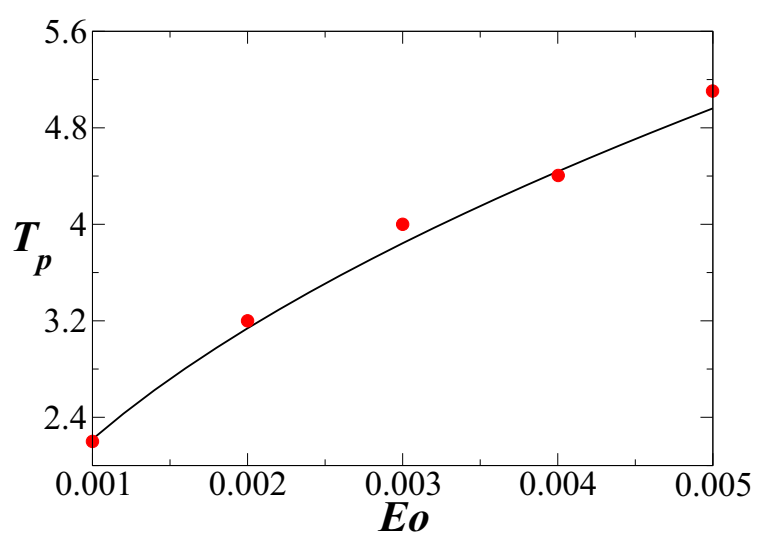

FIG. 9. The dimensionless time period of the oscillations, $T_{p}$ versus Eo. The results from the numerical simulations and theoretical analysis [obtained from Eq. (11)] are shown by symbols and a solid line, respectively. The rest of the parameter values are the same as those used to generate Fig. 8.

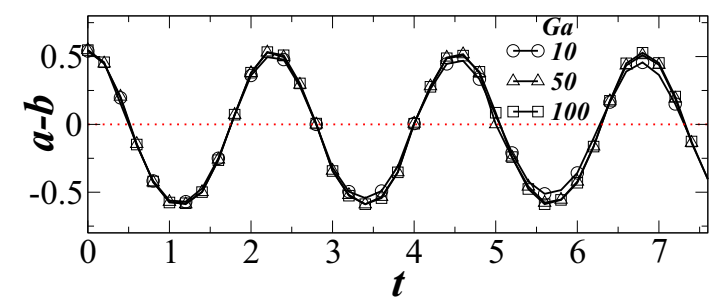

FIG. 10. The temporal variations of $(a-b)$ for $A_{r 0}=1.331$ (Eo $=10^{-3}$ and $\theta=45^{\circ}$ ) for different values of Ga.

Increasing surface tension, i.e., decreasing the value of Eo, brings the droplet faster to a spherical shape from an initially oblate shape (say) and thereby decreases the time period of shape oscillations. Then the inertia effect comes into play by deforming the droplet to a prolate shape. This behavior can also be observed in the theoretical analysis (see Sec. III A and the following discussion).

Figure 9 presents the dimensionless time period of oscillations, $T_{p}$, obtained from the numerical computations for different values of Eo. The theoretically obtained variation of $T_{p}$ [using Eq. (11)] with Eo is also shown, which agrees well with the numerically obtained $T_{p}$ values (shown by symbols in Fig. 9) for different values of Eo. Oscillations of the drop are analogous to the oscillations of an underdamped spring-massdamper system. The natural oscillation frequency $\left(\omega_{n}\right)$ for an undamped spring-mass-damper system is $\sqrt{k / m}$, wherein $k$ and $m$ are the spring constant and the magnitude of the oscillating mass. For a drop, this expression may be compared with Eq. (A11), which implies a resemblance of surface tension coefficient with the spring constant.

The effect of Ga on the droplet dynamics is investigated in Fig. 10 by plotting the temporal variations of $(a-b)$ for (a)

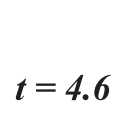

5.0

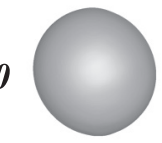

5.6
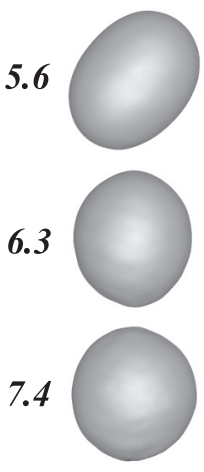

(b)
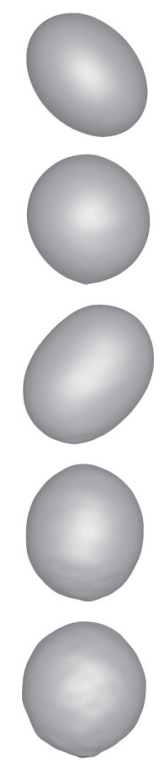

FIG. 11. Evolutions of the shape of the droplet ( $y-z$ view) for $\theta=45^{\circ}$ : (a) $\mathrm{Ga}=300$ and (b) $\mathrm{Ga}=500$. The remaining parameter values are Eo $=10^{-3}$ and $A_{r 0}=1.331$. 


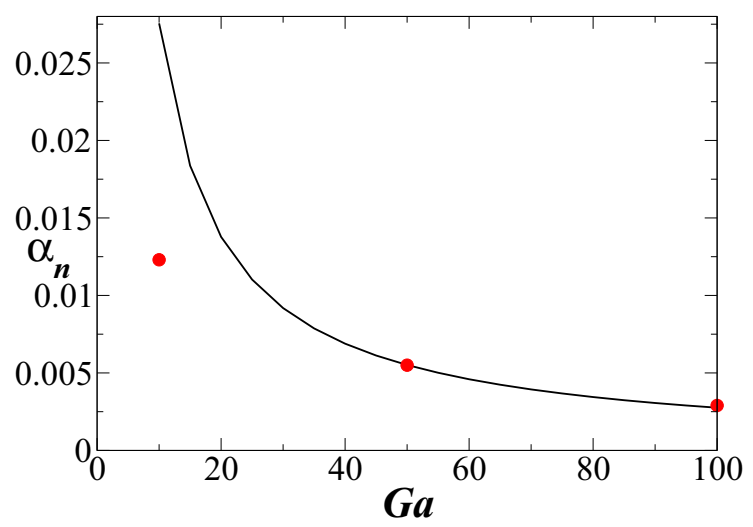

FIG. 12. The dimensionless decay rate of the oscillations, $\alpha_{n}$, obtained from the theoretical analysis [Eq. (12)] versus Ga (solid line). The results from the numerical simulations are shown by symbols. The rest of the parameter values are the same as those used to generate Fig. 10.

$\mathrm{Ga}=10,50$, and 100 . The rest of the parameter values are $A_{r 0}=1.331$, Eo $=10^{-3}$, and $\theta=45^{\circ}$. It can be seen that the droplet undergoes periodic deformation with a dimensionless time period, $T_{p} \approx 2.2$ for $\mathrm{Ga}=10-100$. However, we observe that the amplitude of oscillations increases slightly at later times for $\mathrm{Ga}=100$. Agarwal et al. [14] also showed that the amplitude of oscillations increases with time for high values of Ga. Thus, we may expect nonlinear effects to arise if we increase the value of $\mathrm{Ga}$ further. In order to confirm this, we perform simulations for $\mathrm{Ga}=300$ and 500 and the results are presented in Fig. 11. It is found that the droplet undergoes one cycle of shape oscillation (see the droplet shapes to $t=$ 5.6 in Fig. 11). At later times, we see the appearance of small wavelike structures on the surface of the droplet, which grow and lead to the fragmentation of the droplet.

The dimensionless decay rates for the unbroken droplets, i.e., for $\mathrm{Ga}=10-100$, obtained from the theoretical analysis [Eq. (12)] and the numerical simulations for different values of $\mathrm{Ga}$ are shown in Fig. 12. It can be seen that the dimensionless decay rate of oscillations, $\alpha_{n}$, decreases with increasing $\mathrm{Ga}$. For $\mathrm{Ga}=50$ and 100 , the results from the theoretical analysis and numerical simulations agree well; however, we observe some deviation in the value of $\alpha_{n}$ for $\mathrm{Ga}=10$. Note that in Eq. (12), the value of $\alpha_{n}$ only depends on Ga. In the inviscid limit $(\mathrm{Ga} \rightarrow \infty)$, it can be seen in Eq. (12) that $\alpha_{n} \rightarrow$ 0 [12]. However, in our nonlinear simulations for $\mathrm{Ga}=300$ and 500, as expected, the droplet undergoes fragmentation. It is observed (not shown) that the droplet breaks at later times for $\mathrm{Ga}=300$ and $\mathrm{Ga}=500$.

\section{CONCLUDING REMARKS}

The dynamics of a nonspherical droplet falling in air is investigated via three-dimensional numerical simulations using an open-source VoF-based multiphase flow solver, Basilisk [20,21]. The dimensionless parameters considered in this study are similar to those of a typical falling raindrop. A grid convergence test is conducted to ensure that we have used adequate numbers of cells to capture the physics. The effects of initial aspect ratio, $A_{r 0}$, orientation of the droplet, $\theta$, the
Eötvös number, Eo, and the Galilei number, Ga, are investigated. The frequency of shape oscillations obtained from our numerical simulations agrees well with the theoretical prediction under the creeping flow approximation and also the experimental result of Szakall et al. [3].

We found that the orientation of the droplet has a negligible effect on shape oscillations; however, there are changes in the vortex shedding patterns. Symmetrical vortex shedding is observed for $\theta=0^{\circ}$ and $90^{\circ}$, whereas countersymmetry vortex shedding occurs for other $\theta$ values considered. Increasing the value of Eo for the $45^{\circ}$-oriented droplet increases the time period of oscillations, which is also in agreement with the theoretical prediction.

Finally, the effect of $\mathrm{Ga}$ on droplet dynamics is studied. Although the effect of Ga has been investigated [14], the point we would like to make here is that the amplitude of oscillations for the parameter range observed in raindrops is either constant or increases with time. However, the dimensionless decay rate of these oscillations for this set of parameters is very small $\left(\alpha_{n} \approx 3 \times 10^{-3}\right.$ for $\left.\mathrm{Ga}=100\right)$. In other words, a raindrop would continue to exhibit the shape oscillations until it hits the ground (the typical altitude of cloud is 1 to $2 \mathrm{~km}$ ). We remark that the periodic shape oscillations observed in the present study would influence coalescence and breakup phenomena during rain, which will be an interesting topic to study in the future.

\section{ACKNOWLEDGMENT}

K.C.S. thanks DST - MATRICS India for the financial support through the Grant No. MTR/2017/000029.

\section{APPENDIX: THEORETICAL PREDICTION OF SHAPE OSCILLATIONS}

Here we derive the expressions of the dimensional frequency and decay rate of oscillations of an axisymmetric droplet, whose surface is given by

$$
r_{s}(\phi, t)=R_{\mathrm{eq}}+A_{n}(t) P_{n}(\cos \phi)
$$

in microgravity condition. This analysis is similar to that given in Lamb [9] and Tsamopoulos and Brown [10]. A spherical coordinate system is used, such that $\phi$ is the polar angle, $t$ is time, $P_{n}$ is a Legendre polynomial of degree $n$, and $A_{n}$ is the time-dependent displacement function. Equation (A1) can be written in the general form by the superposition of spherical harmonics or modes as

$$
r_{s}(\theta, t)=R_{\text {eq }}\left[1+\sum_{n=0}^{\infty} A_{n}(t) P_{n}(\cos \theta)\right] .
$$

Here $A_{n}$ is assumed to be small such that the nonlinear effect is neglected. As the flow is assumed to be incompressible and irrotational, $\nabla^{2} \Phi=0$, where $\Phi$ is the velocity potential. The solution of $\Phi$ is given by

$$
\Phi=B_{n}(t)\left(\frac{r}{R_{\mathrm{eq}}}\right)^{n} P_{n}(\cos \phi) .
$$


Substituting $\Phi$ and $r_{s}$ in the linearized kinematic boundary condition (i.e., $\partial r_{s} / \partial t=\partial \Phi / \partial r$ ) at the droplet surface, we get

$$
\frac{d A_{n}}{d t}=\frac{n B_{n}}{R_{\mathrm{eq}}} .
$$

By taking the dot product of the momentum conservation equations with the velocity vector, $\nabla \Phi$, and then integrating the resultant equation over the volume of the droplet, $\mathcal{V}$, we get the following energy balance equation:

$$
\frac{d E}{d t}=W-D
$$

where $W$ is the rate of surface work done, given by $W=\int_{S} \mathbf{S}$. $(\nabla \Phi) d s$ and $E$ is the kinetic energy of the droplet, which is given by $E=\int_{\mathcal{V}} \rho(\nabla \Phi) \cdot(\nabla \Phi) d \mathcal{V} / 2$. This can be written as

$$
E=\left(\frac{2 \pi n}{2 n+1}\right) \rho_{i} R_{\mathrm{eq}} B_{n}{ }^{2} .
$$

Here $\mathbf{S}$ is the total stress at the surface, which is given by $\mathbf{S}=(-p+\sigma \kappa) \mathbf{n}$, wherein $p$ is the pressure field and $\kappa$ is the interfacial curvature. For the surface given by Eq. (A1), the curvature is

$$
\kappa=-\left[\frac{2}{R_{\mathrm{eq}}}+\frac{(n-1)(n+2)}{R_{\mathrm{eq}}^{2}} A_{n} P_{n}(\cos \phi)\right] .
$$

Using the above expressions of $\kappa$ and $\mathbf{S}$, the rate of surface work done $W$ is given by

$$
W=-\left(\frac{4 \pi}{2 n+1}\right) n(n-1)(n+2)\left(\frac{\sigma}{R_{\mathrm{eq}}}\right) A_{n} B_{n} .
$$

The rate of viscous dissipation, $D=\int_{\mathcal{V}} 2 \mu_{i} \epsilon_{i j} \epsilon_{i j} d \mathcal{V}$. This can also be expressed as

$$
D=8 \pi n(n-1)\left(\frac{\mu_{i}}{R_{\mathrm{eq}}}\right) B_{n}^{2} .
$$

Using Eqs. (A6), (A8), and (A9) in Eq. (A5), we get

$$
\frac{d^{2} A_{n}}{d t^{2}}+2 \alpha_{n, d} \frac{d A_{n}}{d t}+\omega_{n, d}^{2} A_{n}=0
$$

This equation is similar to that describes the damped vibration of a spring-mass-damper system. Here $\omega_{n, d}$ and $\alpha_{n, d}$ are the dimensional frequency and decay rate of the oscillations. They are given by

$$
\omega_{n, d}=\sqrt{n(n-1)(n+2) \frac{\sigma}{\rho_{i} R_{\mathrm{eq}}^{3}}}
$$

and

$$
\alpha_{n, d}=(n-1)(2 n+1) \frac{\mu_{i}}{\rho_{i} R_{\mathrm{eq}}^{2}}
$$

[1] H. R. Pruppacher and R. Pitter, J. Atmos. Sci. 28, 86 (1971).

[2] K. V. Beard, H. T. Ochs III, and R. J. Kubesh, Nature 342, 408 (1989).

[3] M. Szakall, D. Karoline, S. K. Mitra, and S. Borrmann, J. Atmos. Sci. 66, 755 (2009).

[4] M. Thurai, V. N. Bringi, M. Szakáll, S. K. Mitra, K. V. Beard, and S. Borrmann, J. Atmos. Ocean. Technol. 26, 1427 (2009).

[5] E. Villermaux and B. Bossa, Nat. Phys. 5, 697 (2009).

[6] B. K. Jones and J. R. Saylor, J. Atmos. Ocean. Technol. 26, 2413 (2009).

[7] J. Saylor and B. Jones, Phys. Fluids 17, 031706 (2005).

[8] H. R. Pruppacher and J. D. Klett, Microphysics of Clouds and Precipitation (Springer, Dordrecht, 2010).

[9] H. Lamb, Hydrodynamics, 6th ed. (Cambridge University Press, Cambridge, 1993).

[10] J. A. Tsamopoulos and R. A. Brown, J. Fluid Mech. 127, 519 (1983).

[11] A. Salih and M. S. Ghosh, in Proceedings of the 2nd International Conference on Fluid Mechanics and Fluid Power, Vol. 1025 (2002).
[12] L. Rayleigh, Proc. R. Soc. Lond. 29, 71 (1879).

[13] J. Q. Feng and K. V. Beard, J. Atmos. Sci. 48, 1856 (1991).

[14] M. Agrawal, A. R. Premlata, M. K. Tripathi, B. Karri, and K. C. Sahu, Phys. Rev. E 95, 033111 (2017).

[15] C. J. Koh and L. G. Leal, Phys. Fluids A 2, 2103 (1990).

[16] C. J. Koh and L. G. Leal, Phys. Fluids A 1, 1309 (1989).

[17] D. V. Svintradze, arXiv:1802.07477.

[18] R. M. Edge and C. D. Grant, Chem. Eng. Sci. 26, 1001 (1971).

[19] C. Miller and L. Scriven, J. Fluid Mech. 32, 417 (1968).

[20] S. Popinet, J. Comput. Phys. 190, 572 (2003).

[21] S. Popinet, J. Comput. Phys. 228, 5838 (2009).

[22] M. K. Tripathi, K. C. Sahu, and R. Govindarajan, Nat. Commun. 6, 6268 (2015).

[23] M. K. Tripathi, K. C. Sahu, and R. Govindarajan, Sci. Rep. 4, 4771 (2014).

[24] M. K. Tripathi, K. C. Sahu, G. Karapetsas, K. Sefiane, and O. K. Matar, J. Fluid Mech. 763, 82 (2015).

[25] M. K. Yau and R. R. Rogers, A Short Course in Cloud Physics (Elsevier, Amsterdam, 1996). 